\begin{tabular}{lllll}
$\begin{array}{l}\text { 12-17 year- } \\
\text { olds }\end{array}$ & 3171 & $\begin{array}{l}0.70(0.57, \\
0.86)\end{array}$ & 241 & $0.67(0.54$, \\
$\begin{array}{l}\text { Adult } \\
\text { Controls }\end{array}$ & 4543 & - & 361 & - \\
\hline
\end{tabular}

Conclusion Based on these results, children aged 2-11 yrs require a higher $\mathrm{mg} / \mathrm{kg} /$ day dose of rofecoxib for similar steadystate systemic exposure to adult historical controls receiving 25 mg. Assuming linear dose proportionality, a dose of $\sim 0.6 \mathrm{mg} / \mathrm{kg} /$ day in children aged 2-11 yrs, should approximate the exposure of $25 \mathrm{mg}$ in adults and adolescents aged 12-17 yrs showed similar systemic exposure to adults taking identical doses of rofecoxib.

Funded by a grant from Merck and Co., Inc.

\section{OP0095 THERAPEUTIC EFFICACY OF MULTIPLE INTRAVENOUS INFUSIONS OF ANTI-TUMOUR NECROSIS FACTOR-ALFA MONOCLONAL ANTIBODY (INFLIXIMAB) IN PERSISTENTLY ACTIVE REFRACTORY JUVENILE IDIOPATHIC (CHRONIC) ARTHRITIS. AN ONE YEAR OPEN PROSPECTIVE PILOT STUDY}

V Gerloni, I Pontikaki, F Desiati, E Lupi, M Gattinara, F Fantini. Chair of Rheumatology of the University of Milan, Centre for Rheumatic Children, Gaetano Pini Institute, Milano, Italy

\subsection{6/annrheumdis-2001.497}

\section{Background}

Objectives An open prospective trial was carried out in a young population affected by severe, refractory, polyarthritic JIA to evaluate the efficacy and safety of Infliximab (Remicade).

Methods We enrolled 17 females, median age at the start of the therapy 22.9 yrs (8-33), median onset age 3 yrs (1-16), median disease duration 15.6 yrs (5-31). All patients had been previously treated with more than one DMARD (median number 3.5/ patient, as mono or combined therapy). All patients had still active disease: number of active joints (median 5.5), ESR (median $59.5 \mathrm{~mm} / \mathrm{hr}$ ), CRP (median $4.7 \mathrm{mg} / \mathrm{dl}$ ), physician global evaluation (median VAS 45.5), HAQ (median D. I. 1.13). 14 patients were still on corticosteroids (median $0.11 \mathrm{mg} / \mathrm{kg}$ ). All patients discontinued any other DMARD aside from s.c. MTX (median weekly dose $15 \mathrm{mg}$, range 5-25) and continued with previous NSAIDs and corticosteroids. Infliximab was administrated as a single infusion of $3 \mathrm{mg} / \mathrm{kg}$ at time 0 and at weeks 2 , $6,14,22,30$.

Results Until now all 17 pts have received the first 2 infusions, 15 pts 3 infusions, 9 pts 6 infusions, 8 pts 7 infusions, 2 pts 8 infusions and 1 pt 10 infusions. One patient withdrew because of a severe adverse event (hypersensitivity reaction at the third infusion). Since the 1 st infusion, all 17 patients achieved a very good response $(50 \%$ reduction in number of active joints, ESR, CRP, VAS). A statistically significant improvement of the following parameters was observed at the 6th infusion assessment (evaluation on 9 patients who completed 30 weeks $=5$ months of therapy): the median number of active joints decreased from 5 to 0; ESR decreased from a median of 65 to $23 \mathrm{~mm} / \mathrm{hr}$ and CRP decreased from a median of 4.6 to $0.4 \mathrm{mg} / \mathrm{dl}$; the median score of the VAS (physician global evaluation) decreased from a 65 to 2. Furthermore after 6 months of therapy we observed a trend to improvement in all scales of SF36 (assessment of the quality of life). An eleven-year-old girl with chronic active uveitis showed a great improvement of the visus.
Conclusion These data suggest that Infliximab can significantly and promptly reduce disease activity and improve the quality of life of patients affected by persistently active and refractory JIA. However more data are needed to evaluate its efficacy and safety as a long-term therapy.

\section{OP0099 MOOD AND STRESSFUL EVENTS PREDICT DAILY FLUCTUATIONS IN DISEASE SYMPTOMS IN JUVENILE POLYARTICULAR ARTHRITIS}

${ }^{1}$ LE Schanberg, ${ }^{2} \mathrm{KM}$ Gil, ${ }^{2} \mathrm{KK}$ Anthony, ${ }^{2} \mathrm{E}$ Maurin. ${ }^{1}$ Pediatrics, Duke University Medical Center, Durham, USA; ${ }^{2}$ Psychology, University of North Carolina, Chapel Hill, USA

\subsection{6/annrheumdis-2001.498}

Background Juvenile arthritis is characterised by periods of flare and relative quiescence. Periods of flare are characterised by increased disease activity and worsening symptoms. While the cause of disease flares is unclear, multiple possible triggers have been proposed including infection, trauma, psychosocial stress, and mood.

Objectives The purpose of this study was to examine the relationship of daily mood and daily stressful events to disease symptoms in children with rheumatic disease.

Methods Twenty-four school-aged children (15 girls, 9 boys, mean age 12.4 years) with polyarticular arthritis completed a daily booklet over a two-month period, yielding 1355 total observations. The daily booklet included measures of daily symptoms, function, mood, and stressful events. In addition, we obtained biological measures of disease activity, as well as salivary cortisol levels three times daily. Data were analysed using multi-level random effects modelling in order to take into account both sources of variation in the data set, within-person and between-person. In addition, multilevel models use the number of observations or days rather than the number of participants as the unit of analysis, thus increasing the statistical power. Results The models indicated that on a day-to-day basis, worse mood and more stressful events were significantly related to increases in daily pain $(\mathrm{p}<.0001$ and $\mathrm{p}<.02)$, fatigue $(\mathrm{p}<$. 0001 and $\mathrm{p}<.02$ ), and increased interference in social activities $(\mathrm{p}<.0001$ and $\mathrm{p}<.001)$. Worse mood was also related to increases in daily stiffness $(\mathrm{p}<.0001)$ and to coping, including the ability to control $(\mathrm{p}<.0001)$ and decrease pain $(\mathrm{p}<$. 0001).

Conclusion Our data show that day to day fluctuations in mood and stressful events are related to the daily symptoms of children with juvenile polyarticular arthritis and may play a role in the pathogenesis of disease flares. Therefore, interventions aimed at improving mood and stress coping may improve the health status of children with arthritis.

\section{SAT0106 ANTI-TNF-ALPHA THERAPY FOR SYSTEMIC JUVENILE IDIOPATHIC ARHTRITIS WITH POLYARTHRITIS}

${ }^{1} \mathrm{C}$ Wouters, ${ }^{2} \mathrm{~F}$ Cornillie, ${ }^{1} \mathrm{AD}$ Billiau. 'Paediatric Rheumatology, UZ Gasthuisberg, Catholic University of Leuven, Leuven, Belgium; ${ }^{2}$ Immunology Clinical Information Services, Centocor $B V$, Leuven, Belgium

\subsection{6/annrheumdis-2001.499}

Background TNF- $\alpha$ antagonism with anti-TNF- $\alpha$ MoAb (Infliximab, Centocor, USA) or soluble TNF- $\alpha$ receptors (Etanercept, Immunex, USA), has emerged as a promising therapeutic tool for refractory chronic arthritis. Both drugs are now licensed for 
the treatment of refractory RA but experience with TNF- $\alpha$ antagonisers in JIA is limited.

Objectives We report the use of Infliximab in 3 patients with systemic JIA and polyarthritis.

Methods LS (18 yrs) and JD (11 yrs), girls with systemic-onset JIA at the age of 11 and 3, had persistent, destructive polyarthritis. AP (10 yrs), a boy with systemic-onset JIA at the age of 5 had persistent systemic activity as well as severe polyarthritis. All 3 failed NSAID, corticosteroids, (high-dose) MTX and CsA treatment and suffered from severe growth impairment and osteoporosis. All patients started Infliximab therapy at $3 \mathrm{mg} / \mathrm{kg}$ (weeks 0, 2, 6 and then 8-weekly). Active joint number (AJN), limited joint number (LJN), visual analogue scale for global well being (VASg) and pain (VASp), Childhood Health Assessment Questionnaire (CHAQ), serum CRP and serum IL-6 were assessed.

Results At present, LS completed 26, JD 38 and AP 86 treatment weeks. LS experienced a manifest decrease in AJN (17 to 1). LJN was unchanged (8). Functional scores decreased markedly: VASg 78 to 10 , VASp 45 to 10 , CHAQ 1.2 to 0.3 . CRP decreased ( 15 to $3 \mathrm{mg} / \mathrm{l}$ ) and steroids were tapered from 6 to 3 mg daily. JD showed a similar response: AJN and LJN decreased rapidly (38 to 5 and 41 to 7 ). VASg, VASp and CHAQ all dropped markedly: 50 to 15,50 to 10 and 2.1 to 1.4 . CRP and ESR decreased ( 22 to $5 \mathrm{mg} / \mathrm{L}$ and 51 to $26 \mathrm{~mm} / \mathrm{hr}$ ). Steroid dose was kept at $4 \mathrm{mg}$ daily. AP experienced a decrease in AJN (9 to 3 ) and in LJN (12 to 4 ) during the first 18 weeks. As systemic inflammation persisted, Infliximab dose was increased to 5, later to $10 \mathrm{mg} / \mathrm{kg}$. Whereas AJN and LJN decreased further to 0 and 2, systemic symptoms and the acute phase response persisted: CRP and ESR fluctuated (15-148 mg/l and 45 ? $183 \mathrm{~mm} / \mathrm{h}$, respectively). Functional scores varied and reflected the increasing discomfort of preexisting joint damage. Steroid dose was tapered from 12 to $7 \mathrm{mg}$ daily. Infliximab was safe and well tolerated in all 3 patients: possible adverse reactions were fever (AP, once) and upper respiratory tract infection (AP three, JD once). TNF- $\alpha$ blockade with Infliximab in these patients with systemic-onset polyarticular JIA induced a rapid, sustained control of polyarthritis, with concomitant improvement of function, global well being and pain and decrease in biochemical inflammation. Systemic inflammation, however, did not respond to TNF- $\alpha$ blockade. This was evident in patient AP whose articular disease clearly responded but who had a persistent acute phase response with persistently elevated levels of CRP and IL-6.

Conclusion Infliximab is promising for the treatment of JIA. The observed dissociation between response of articular and systemic disease indicates that different cytokine networks may operate in systemic and articular inflammation of JIA.

\section{SAT0107 YOUNG ADULTS WITH JUVENILE ARTHRITIS (JA) IN REMISSION ATTAIN IMPROVED NUTRITIONAL STATUS COMPARED WITH HEALTHY CONTROLS}

M Haugen, G Lien, B Flatø, Ø Førre. Center for Rheumatic Diseases, The National Hospital, Oslo, Norway

10.1136/annrheumdis-2001.500

\section{Background}

Objectives The purpose of the study was to compare nutritional status in adults with a history of JA with nutritional status in healthy subjects.
Methods All patients diagnosed with JA between 1980-1985 were re-investigated in 1997. Eighty-eight female patients and 51 male patients were $>20$ years of age and in remission at the time-point of investigation. Mean age was $25.2 \pm 2.8$ years in females and $25.0 \pm 3.0$ years in males. Mean age at disease debut was $9.7 \pm 3.2$ in the females and $10.1 \pm 3.4$ in the males. Median years (range) with active disease was 6.7 (0.3-25.1) in the female group and $3.3(0.1-15.5)$ in the male group. Fortyone healthy females (mean age $27.4 \pm 3.1$ years) and 54 healthy males (mean age $25.7 \pm 3.1$ years) were recruited from a military base in the Oslo area as controls. Total body lean body mass and body fat percentages were measured with DXA scanner (Lunar Expert). As laboratory measurements serum albumin concentration and haemoglobin concentrations were measured. Dietary intake was evaluated from a quantitative food frequency questionnaire.

Results While Body Mass Index (weight/height2) was similar in the two groups the subjects with a history of JA had significantly more lean mass than the healthy controls $(p<0.01$ for both females and males) and had significantly lower percentage of body fat ( $\mathrm{p}<0.001$ in both females and males). The subjects with a history of JA had a statistical significant higher concentration of haemoglobin $(p<0.001$ for the females and $p<0.05$ for the males) and serum albumin concentration ( $p<0.001$ for both) compared with the healthy controls. The patient groups had a higher energy intake per kilo body weight and fat intake than the control group, though this was only statistical significant in the male group $(\mathrm{p}<0,05)$. The frequency and amount of physical activity at the time-point of investigation was similar in the patients with a history of JA and the controls.

Conclusion Nutritional status in young adults with a history of JA does not seem to affected by disease during puberty, at least not negatively.

\section{SAT0108 UVEITIS AND ITS OUTCOME IN PATIENTS WITH JUVENILE IDIOPATHIC ARTHRITIS - LONG TERM STUDY}

${ }^{1} \mathrm{~K}$ Jarosova, ${ }^{2} \mathrm{D}$ Nemcova, ${ }^{1} \mathrm{~J}$ Vencovsky. ${ }^{1}$ Institute of Rheumatology; ${ }^{2}$ Department of Pediatrics, Charles University, Prague, Czech Republic

10.1136/annrheumdis-2001.501

\section{Background}

Objectives To determine the frequency of uveitis and its complications in patients with juvenile idiopathic arthritis (JIA).

Methods A retrospective analysis of 185 patients with JIA diagnosed between 1980 ? 1995 followed in 2 paediatric rheumatology centres. Patients with chronic and acute uveitis were identified in subgroups of JIA.

Results Uveitis was diagnosed in $24(13.0 \%)$ patients. Sixteen (8.6\%) patients had chronic and eight $(4.3 \%)$ had acute uveitis. Twelve $(75.0 \%)$ patients who developed chronic uveitis did so after the onset of arthritis, but only 8 within the first 7 years of JIA. There were four patients who developed chronic uveitis 9, 13, 14 and 17 years after JIA onset. Four patients who manifested arthritis after uveitis did so after $1,3,3$ and 6 years. Chronic uveitis was associated with systemic arthritis (in 17.6\%), persistent oligoarthritis (in 29.6\%), extended oligoarthritis (in 4,5\%), psoriatic arthritis (in 40\%) and other arthritis (in 14.3\%). Complications, such as synechiae, band keratopathy, cataract or glaucoma developed in $10(62.5 \%)$ patients with chronic uveitis. As expected, these were found mainly in patients with ANA positivity ( $\mathrm{n}=8 ; 80.0 \%$ ), in contrast to only 2 between ANA 